VOL. 60 (1999) [487-493]

\title{
ON THE THREE-SPACE PROBLEM FOR THE DUNFORD-PETTIS PROPERTY
}

\author{
J.M.F. Castillo and M.A. Simoes
}

\begin{abstract}
A Banach space $X$ is called a twisted sum of the Banach spaces $Y$ and $Z$ if it has a subspace isomorphic to $Y$ in such a way that the corresponding quotient is isomorphic to $Z$. In this paper we study twisted sums of Banach spaces with either have the Dunford-Pettis property, are $c_{0}$-saturated or $l_{1}$-saturated. Amongst other things, we show that every Banach space is a complemented subspace of a twisted sum of two Banach spaces with the Dunford-Pettis property.
\end{abstract}

\section{INTRODUCTION}

Let $\mathcal{P}$ and $\mathcal{Q}$ be two properties of Banach spaces stable by isomorphisms. Following [7], a Banach space $X$ is said to have the $\mathcal{P}$-by- $\mathcal{Q}$ property if it admits a subspace $Y$ with property $\mathcal{P}$ so that $X / Y$ has property $\mathcal{Q}$. In such case, we shall also say that $X$ is a twisted sum of $Y$ and $Z$ (in this order). If $Y$ is complemented in $X$ we say that it is a trivial twisted sum. A property $\mathcal{P}$ is said to be a three-space property (3-space property in short) if $\mathcal{P}$-by- $\mathcal{P}$ implies $\mathcal{P}$. The monograph [5] contains rather complete information about 3-space problems in Banach spaces.

In the present paper we are interested in the choices

$$
\mathcal{P}, \mathcal{Q} \in\left\{\text { Dunford-Pettis, } c_{0} \text {-saturation, } l_{1} \text {-saturation }\right\}
$$

Previously known results are that the Dunford-Pettis property is not a 3-space property, while $c_{0}$-saturation, $l_{1}$-saturation and the Schur property are well-known 3-space properties (see [5]). Let us briefly recall the meaning of those properties. The DunfordPettis property (DPP for short) means that given two weakly null sequences $\left(x_{n}\right)$ and $\left(f_{n}\right)$ in $X$ and $X^{*}$, respectively, then $\lim _{n} f_{n}\left(x_{n}\right)=0$. It is an important property of Banach spaces, notwithstanding the fact that very few examples of spaces with this property are known. The main examples are the $C(K)$ and $L_{1}(\mu)$-spaces; or, more generally, the $\mathcal{L}_{\infty}$

Received 8th March, 1999

The research of the first author has been supported in part by DGICYT project PB97-0377. It is a pleasure to thank Félix Cabello Sánchez who suggested the possibility of obtaining Proposition 4; and David Yost who remarked that Proposition 1 should be as it is.

Copyright Clearance Centre, Inc. Serial-fee code: 0004-9727/99 \$A2.00+0.00. 
and $\mathcal{L}_{1}$ spaces. Even more scarce are those spaces such that every closed subspace has DPP. They are called spaces with the hereditary Dunford-Pettis property $(\mathrm{DPPh}) ; c_{0}(\Gamma)$ and $l_{1}(\Gamma)$ are perhaps the simplest examples. A Banach space $X$ is said to be $c_{0}$-saturated (respectively $l_{1}$-saturated) if every closed subspace contains $c_{0}$ (respectively $l_{1}$ ). We shall study the implications between these notions in section 4 . Now we simply recall that particular cases of DPPh-spaces are the Schur spaces: those in which weakly convergent sequences are norm convergent; that if $X^{*}$ has the DPP then $X$ has the DPP and that if $X$ has the DPP then $X^{*}$ is Schur if and only if $X$ does not contain $l_{1}$.

\section{The tWisted Dunford-PetTis PROPERTy}

In [6] it was proved that the Dunford-Pettis property is not a 3-space property, (this was an open problem for some time). The following result shows that the world has plenty of counterexamples.

THEOREM 1. Every Banach space is a complemented subspace of a twisted sum of two Banach spaces with the Dunford-Pettis property.

ProOF: Let $X$ be a Banach space. It is possible to find a compact space $K$ such that $X$ is isomorphic to a subspace of $C(K)$ and then an index set $\Gamma$ such that $C(K)$ is isomorphic to a quotient of $l_{1}(\Gamma)$. Let $j: X \rightarrow C(K)$ be the isomorphic embedding and $Q: l_{1}(\Gamma) \rightarrow C(K)$ the quotient map. Since $Q^{-1}(j(X))$ is a subspace of $l_{1}(\Gamma)$ it has the Schur property (hence, the hereditary Dunford-Pettis property). If $p \in Q^{-1}(j(X))$ there exists a unique $x \in X$ such that $Q p=j x$. Thus, the operator $T: Q^{-1}(j(X)) \rightarrow l_{1}(\Gamma) \oplus X$ given by $T p=(p, x)$ is well-defined and an isomorphic embedding. Moreover, the operator $l_{1}(\Gamma) \oplus X \rightarrow C(K)$ given by $(l, x) \rightarrow Q l-j x$ is a quotient map whose kernel is precisely $T Q^{-1}(j(X))$.

Hence, it makes sense to define the twisted Dunford-Pettis property as the property $D P P$-by-DPP. Reflexive spaces do not possess the twisted DPP. There exist $c_{0}$-saturated spaces without the twisted Dunford-Pettis property:

Proposition 1. The Schreier space does not have the twisted DPP.

Proof: The Schreier space $S$ (see [16]) is $c_{0}$-saturated, and every quotient of $S$ is $c_{0}$-saturated (see [14]). Thus, if $S$ is the twisted sum of two DPP spaces $Y$ and $Z$ then both $Y^{*}$ and $Z^{*}$ would be Schur spaces. This being a 3 -space property, also $S^{*}$ should be a Schur space, which it is not since it fails the DPP.

It would be interesting to know if the twisted DPP and the DPP coincide for $c_{0}$ saturated spaces; we remark in passing that quotients of $c_{0}$-saturated spaces need not be $c_{0}$-saturated (see [12]). As a corollary to the proof of Theorem 1 , choosing $X=S$ and $C(K)=C\left(\omega^{\omega}\right)$ we obtain:

PROPOSITION 2. Schur-by-(DPP and $c_{0}$-saturated) does not imply DPP. 
This result shall be completed with Propositions 6 and 7. Exchanging the two properties yields (see $[5,6.6 . c]$ ).

Proposition 3. DPP-by-Schur and DPP coincide.

\section{TWisted sums of $c_{0}$-SATURATEd AND $l_{1}$-SATURATEd SPACES}

In $[5,6.6 . d$.$] , it was proved that the DPPh is equivalent to the property that every$ weakly null sequence admits a weakly 1-summable subsequence, that is, a subsequence satisfying an estimate

$$
\sup _{F \in F I N(\mathbb{N})}\left\|\sum_{n \in F} \pm x_{n}\right\|<+\infty
$$

where $F I N(\mathbb{N})$ denotes the set of finite subsets of $\mathbb{N}$. We say that a Banach space is $\left\{c_{0}, l_{1}\right\}$-saturated if every closed infinite dimensional subspace contains either $c_{o}$ or $l_{1}$. It is clear that Banach spaces with the DPPh are $\left\{c_{0}, l_{1}\right\}$-saturated. Our first result clarifies the structure of twisted sums of $c_{0}$-saturated and $l_{1}$-saturated spaces.

Propos I TION 4. (co-saturated)-by-( $l_{1}$-saturated) implies $\left\{c_{0}, l_{1}\right\}$-saturated; also ( $l_{1}$-saturated)-by-( $c_{0}$-saturated) implies $\left\{c_{0}, l_{1}\right\}$ saturated.

ProOF: Let $X$ be a twisted sum of a $c_{0}$-saturated space $Y_{0}$ and a $l_{1}$-saturated space $Z_{1}$. Let $W$ be a subspace of $X$ that does not contain $c_{0}$. Since $Y_{0}$ and $W$ are totally incomparable, $Y_{0}+W$ is closed (see [15]) in $X$; therefore $W$, which is isomorphic to $W /\left(W \cap Y_{0}\right)$, is also isomorphic to $\left(Y_{0}+W\right) / Y_{0}$; this is a subspace of $Z_{1}$, and hence $l_{1}$-saturated. The proof of the other case is entirely analogous and thus we omit it.

We show that the converse does not hold. Our counterexample is modelled upon a transfinite version of Schreier space.

Proposition 5. DPPh does not imply ( $c_{0}$-saturated)-by-( $l_{1}$-saturated).

Proof: Let $\omega_{1}$ be the first uncountable ordinal. We say that an element $g \in \omega_{1}^{\omega_{1}}$, that is, a function $g: \omega_{1} \rightarrow \omega_{1}$, is increasing if for every countable ordinal $\alpha \in \omega_{1}, \alpha<$ $g(\alpha)$ and whenever $\alpha<\beta$ then $g(\alpha) \leqslant g(\beta)$. Given an increasing element $g$, a countable subset $A$ of the ordinal interval $\left[0, \omega_{1}\right)$ is said to be $g$-admissible if $\sup A \leqslant g(\min A)$.

We define the Schreier space $S_{g}$ as the closure of the elements of $\mathbb{R}^{\omega_{1}}$ having countable support with respect to the norm

$$
\|x\|_{g}=\sup \left\{\sum_{\alpha \in A}\left|x_{\alpha}\right|: A \text { is } g \text {-admissible }\right\} .
$$

The space $S_{g}$ has the DPPh. Let $\left(x_{n}\right)$ be a weakly null sequence in $S_{g}$. If $\alpha<\omega_{1}$, we denote by $S_{g}(\alpha)$ the subspace formed by those elements having support contained in $[0, \alpha)$. Since elements in $S_{g}$ have countable support and $\omega_{1}$ does not admit countable cofinal subsets, there is some $\Omega<\omega_{1}$, such that $\left(x_{n}\right) \subset S(\Omega)$. The vectors $\left(e_{\gamma}\right)_{\gamma<\Omega}$ form 
a basis for $S(\Omega)$ and thus by standard perturbation arguments the sequence $\left(x_{n}\right)$ can be considered as formed by blocks $\left(u_{n}\right)$ of $\left(e_{\gamma}\right)_{\gamma<\Omega}$. Now, either there is a sequence $\left(n_{j}\right)$ of integers such that if $B_{n}=\operatorname{support}$ of $u_{n}$ then $g\left(\max B_{n_{j}}\right)<\min B_{n_{j+1}}$ for all $j$, or there is no such sequence. If such sequence exists then for every $A \subset \mathbb{N}$ one has $\left\|\sum_{j \in A} u_{n_{j}}\right\|_{g}=1$. If not, there is some index $n_{0}$ and some subsequence $B_{n_{j}} \subset\left(\max B_{n_{0}}, g\left(\max B_{n_{0}}\right)\right]$, in which case $\left\|\sum \lambda_{j} u_{n_{j}}\right\|_{g}=\sum\left|\lambda_{j}\right|$, and one gets a contradiction.

The space $S_{g}$ depends on the choice of the initial function $g$. If $g(\alpha)=\alpha+1$ then $g$-admissible sets are just singletons and $S_{g}=c_{0}\left(\omega_{1}\right)$, while if one could (it is not an increasing function) choose the constant function $g=\omega_{1}$ then $S_{g}=l_{1}\left(\omega_{1}\right)$. In this way, a proper choice of the function $g$, for instance $\omega^{\alpha}<g(\alpha)$, makes some copies of $l_{1}$ appear. In what follows we assume that, for all countable ordinals $\alpha, \omega^{\alpha}<g(\alpha)$. In such case one has:

The space $S_{g}$ is not a twisted sum of a $c_{0}$-saturated space and a $l_{1}$-saturated space. Assume that $S_{g}$ is a twisted sum of a $c_{0}$-saturated space $Y_{0}$ and a $l_{1}$-saturated space $Z_{1}$. Let us first remark that since $S_{g}$ has the DPPh and $Y_{0}$ is $c_{0}$-saturated then $Z_{1}$ has the DPPh (see [5]); being $l_{1}$-saturated, it also has the Schur property.

Now, observe that if $\left(\gamma_{n}\right)$ is a sequence of ordinals such that $g\left(\gamma_{n}\right)<\gamma_{n+1}$ then $\left\|\sum_{n} e_{\gamma_{n}}\right\|_{g}=1$. thus, the sequence $\left(e_{\gamma_{n}}\right)$ is weakly null in $S_{g}$. Therefore $\lim \left\|e_{\gamma_{n}}+Y_{0}\right\|=0$. But this yields that for some $\gamma_{0}<\omega_{1}$ and all $\gamma \geqslant \gamma_{0}$ one has $\left\|e_{\gamma}+Y_{0}\right\|=0$. In this way, $e_{\gamma} \in Y_{0}$ for all $\gamma \geqslant \gamma_{0}$. This is absurd since then $Y_{0}$ would simultaneously be $c_{0}$-saturated and contain $l_{1}$.

REMARK 1. In [8], it is proved that, given a family $\mathcal{F}$ of finite subsets of $\mathbb{N}$ that is compact in the topology of $\{0,1\}^{\mathrm{N}}$, it is possible to construct a (so-called) Schreierlike space $S_{\mathcal{F}}$ that is $c_{0}$-saturated. Some properties of those spaces appear related to properties of a hierarchy of functions $g_{n}$ defined on the finite subsets of $\mathbb{N}$. Precisely, when $S_{\mathcal{F}}$ is an $M$-ideal in its bidual then the Dunford-Pettis and the hereditary Dunford-Pettis properties are equivalent, and equivalent to the finiteness of the function $g_{0}: \mathbb{N} \rightarrow \mathbb{N}$ defined by $g_{0}(n)=\max \{\max A: n \in A \in \mathcal{F}\}$. It is clear that the family $\mathcal{F}$ is then defined as those sets such that $\max A<g_{0}(\min A)$; that is, the Schreier-like space induced by $g_{0}$. The spaces $S_{g}$ can be considered as transfinite constructions of this type where the function $g_{0}$ takes values in $\left[0, \omega_{1}\right)$ instead of in $[0, \omega)$.

Remark 2. Professor Kutzarova has kindly pointed out to us that a type of function like $g_{0}$ was considered in [9] and is at the basis of the construction shown in [10].

REMARK 3. The dependence of $S_{g}$ on the initial choice of $g$ means that there is an uncountable quantity of non-isomorphic $S_{g}$ spaces: let $S_{g}$ and $S_{h}$ be the spaces constructed starting with the functions $g$ and $h$, respectively. Since an isomorphism must transform sequences equivalent to the canonical basis of $c_{0}$ (respectively $l_{1}$ ) into the same class of sequences, a necessary condition to be isomorphic is that, for some $M \in \mathbb{N}$, all $n \in \mathbb{N}$ 
and all countable ordinals $\alpha$

$$
h\left(g^{n}(\alpha)\right) \leqslant g^{n+M}(\alpha) .
$$

The rest is easy since there are uncountably many ways to produce functions not satisfying that requirement; say, for ever ordinal $\beta$ define by transfinite induction functions such that

$$
h_{\beta}\left(h_{\alpha}^{n}(\omega)\right) \leqslant h_{\alpha}^{n+\beta}(\omega)
$$

\section{FURTHER EXAMPLES}

A co-saturated space need not have the DPP: the Schreier space (see [16]) is $c_{0^{-}}$ saturated since it is a subspace of a $C\left(\omega^{\omega}\right)$ and it was shown in [6] that it fails the DPP. A $l_{1}$-saturated space does not necessarily have the $D P P$ either: Lorentz sequence spaces $d(w, 1)$ are $l_{1}$-saturated (see [13, Proposition 4.e.3]) and fail the DPP since they are separable duals without the Schur property.

One might guess that the DPP plus either $c_{0}$ or $l_{1}$-saturation implies the DPPh. There are several examples showing that $c_{0}$-saturated spaces with DPP do not need to have the DPPh; perhaps the simplest example is a $c_{0}$-sum of renormings of $c_{0}$. If $\|\cdot\|_{N}$ denotes the norm

$$
\|x\|_{N}=\sup _{i_{1} \neq i_{2} \neq \cdots \neq i_{N}}\left\{\frac{1}{N} \sum_{j=1}^{N}\left|x_{i_{j}}\right|\right\}
$$

on $c_{0}$ then $X=c_{0}\left(c_{0}, c_{0}\left(\|\cdot\|_{2}\right),, \cdots,, c_{0}\left(\|\cdot\|_{N}\right),, \cdots\right)$ works. By applying [4], this space has the DPP. It is $c_{0}$-saturated since it is a Schreier-like space [8]; or else by [11]. It is not difficult, however, to construct weakly null sequences $\left(r_{n}\right)$ without weakly 1-summable subsequences in $X$ as follows: If $\left\{A_{i}\right\}_{i \in \mathbb{N}}$ is a partition of $\mathbb{N}$ with every $A_{i}$ infinite then $X$ is isomorphic with the completion of the space of finite sequences with respect to the norm

$$
\|x\|=\sup \left\{\sum_{j \in A}\left|x_{j}\right|: A \text { is "admissible" }\right\} ;
$$

where a finite set $A$ is now "admissible" if $A \subset A_{i}$ for some $i$ and $\operatorname{card} A \leqslant i$. Let $\left(B_{n}\right)$ be a sequence of finite subsets of $\mathbb{N}$ such that $\max B_{n}<\min B_{n+1}$. Moreover, the set $B_{n}$ is chosen so that $\operatorname{card} B_{n}=n$, and $\operatorname{card}\left(B_{n} \cap A_{i}\right)=1$, for $1 \leqslant i \leqslant n$. If $r_{n}$ is the characteristic function of $B_{n}$ then $\left\|\sum_{j=1}^{j=N} r_{n_{j}}\right\| \geqslant N / 2$.

Less obvious is the fact that there exist $l_{1}$-saturated spaces with the DPP without the hereditary DPP, as we show now. Bourgain and Pisier show in [1] that given a separable space $E$ it is possible to embed it as a subspace of a certain $\mathcal{L}_{\infty}$-space $\mathcal{L}_{\infty}(E)$ in such a way that $\mathcal{L}_{\infty}(E) / E$ has the Schur property. If we take $E=d(w, 1)$, a suitable Lorentz sequence space (which is $l_{1}$-saturated) with normalised weakly null canonical basis, one obtains a $\mathcal{L}_{\infty}$-space $\mathcal{L}_{\infty}(d(w, 1))$ that is $l_{1}$-saturated, has the DPP but not the DPPh: 
it is $l_{1}$-saturated since this is a 3 -space property [5]; and since in a $l_{1}$-saturated spaces the DPPh and Schur properties are equivalent, it does not have the DPPh because it contains $d(w, 1)$ which is not Schur.

A questions implicit in [5] can be answered now.

PROPOSITION 6. ( $l_{1}$-saturated and DPP) is not a 3-space property.

Proof: The method of Theorem 1, replacing the $C(K)$ space by the $l_{1}$-saturated DPP space $\mathcal{L}_{\infty}(d(w, 1))$ we have just obtained, and the starting space $X$ by $d(w, 1)$, yields that $d(w, 1)$, is a complemented subspace of two ( $l_{1}$-saturated and DPP)-spaces.

Nevertheless, since a Banach space $X$ is ( $c_{0}$-saturated and DPP) if and only if $X^{*}$ is Schur, and the Schur property is a 3-space property, one has.

PROPOSITION 7. (co-saturated and DPP) is a 3-space property.

Proposition 4 is not complete unless one shows it applies to some space. Nontrivial twisted sums of $l_{1}$-saturated and $c_{0}$-saturated spaces are provided by Theorem 1 (let us remark that it is considerably harder, although possible, to show that there exist nontrivial twisted sums of $l_{1}$ and $c_{0}$; see [3] or [2]). Nontrivial twisted sums of $c_{0}$ saturated and $l_{1}$-saturated spaces are, however, harder to find. This is so since the universal property of $l_{1}$ (every surjective map $X \rightarrow l_{1}$ admits a linear continuous section) prevents the existence of nontrivial twisted sums of a Banach space and $l_{1}$; while Sobczyk's Theorem prevents the existence of nontrivial twisted sums of $c_{0}$ and a separable Banach space.

Proposition 8. There exists a separable nontrivial twisted sum of a $c_{0}$ saturated and a $l_{1}$-saturated space.

Proof: Let $E$ be a $c_{0}$-saturated space without the Dunford-Pettis property. There is a separable $\mathcal{L}_{\infty}$-space, in which $E$ cannot be complemented since it lacks the DPP, such that $\mathcal{L}_{\infty}(E) / E$ has the Schur property.

The following question has remained elusive.

Problem 1. Does a $\mathcal{L}_{1}$-by- $\mathcal{L}_{\infty}$-space have the Dunford-Pettis property?

Again, things are simpler when we exchange the properties.

Proposition 9. $\mathcal{L}_{\infty}$-by- $\mathcal{L}_{1}$ implies DPP.

Proof: If $X$ is a twisted sum of a $\mathcal{L}_{\infty}$ space $Y$ and a $\mathcal{L}_{1}$ space $Z$ then $X^{* *}=$ $Y^{* *} \oplus Z^{* *}$ since $Y^{* *}$ is injective. Hence $X^{* *}$ has the DPP and so does $X$.

\section{REFERENCES}

[1] J. Bourgain and G. Pisier, 'A construction of $\mathcal{L}_{\infty}$-spaces and related Banach spaces', Bol. Soc. Brasil Mat. 14 (1983), 109-123. 
[2] Y.A. Brudnyi and N.J. Kalton, 'Polynomial approximation on convex subsets of $\mathbb{R}^{n}$, (preprint).

[3] F. Cabello Sánchez and J.M.F. Castillo, 'Uniform boundedness and twisted sums of Banach spaces', (preprint 37 Uex, 1998).

[4] J.M.F. Castillo and M. González, 'New results on the Dunford-Pettis property', Bull. London Math. Soc. 27 (1995), 599-605.

[5] J.M.F. Castillo and M. González, Three-space problems in Banach space theory, Lecture Notes in Mathematics 1667 (Springer-Verlag, Berlin, Heidelberg, New York, 1997).

[6] J.M.F. Castillo and M. Gonzalez, "The Dunford-Pettis property is not a three-space property', Israel J. Math. 81 (1993), 297-299.

[7] J.M.F. Castillo, M. González, A.M. Plichko and D. Yost, 'Twisted properties of Banach spaces', Math. Scand. (to appear).

[8] J.M.F. Castillo, M. González and F. Sánchez, 'M-ideals of Schreier type and the Dunford-Pettis property', in Non-associative algebra and its applications, (Santos González, Editor), Math. Appl. 303 (Kluwer Acad. Press., Dordrecht), pp. 80-85.

[9] D. Kutzarova, 'On a class of Banach spaces', Math. Ed. 6 (1981), 147-151.

[10] D. Kutzarova and S. Troyanski, 'On reflexive Banach spaces without equivalent norms which are uniformly convex or uniformly differentiable in every direction', Studia Math. 72 (1982), 91-95.

[11] D.H. Leung, 'Some stability properties of $c_{0}$-saturated spaces', Math. Proc. Cambridge Philos. Soc. 118 (1995), 287-301.

[12] D.H. Leung, 'On $c_{0}$-saturated Banach spaces', Mlinois J. Math. 39 (1995), 15-29.

[13] J. Lindenstrauss and L. Tzafriri, Classical Banach spaces I, sequence spaces (Springer-Verlag, Berlin, Heidelberg, New York, 1977).

[14] E. Odell, 'On quotients of Banach spaces having shrinking unconditional bases', Mlinois J. Math. 36 (1992), 681-695.

[15] H.J. Rosenthal, 'On totally incomparable Banach spaces', J. Funct. Anal. 4 (1969), $167-175$.

[16] J. Schreier, 'Ein Gegenbeispiel zur Theorie der swachen Konvergenz', Studia Math. 2 (58-62), 1930.

Departamento de Matemáticas

Universidad de Extremadura

Avenida de Elvas

06071-Badajoz

Spain

e-mail: castillo@unex.es
Dipartimento di Matematica

Universitá di Roma I

"La Sapienza", Piazzale Aldo Moro 2

I-00185 Roma

Italy

e-mail: simoes@mat.uniromal.it 